\title{
Periapical Lesions Increase Macrophage Infiltration and Inflammatory Signaling in Muscle Tissue of Rats
}

\author{
Renato Felipe Pereira, MSc, * Luciano Tavares Angelo Cintra, Prof, ${ }^{\dagger}$ \\ Gestter Willian Lattari Tessarin, MSc, ${ }^{\neq}$Fernando Yamamoto Chiba, PhD, ${ }^{\mathfrak{}}$ \\ Maria Sara de Lima Coutinbo Mattera, MSc, * Natalia Francisco Scaramele, \\ Thais Verônica Saori Tsosura, MSc, "Edilson Ervolino, Prof, \\ Sandra Helena Penha de Oliveira, Prof, ${ }^{\star}$ and Doris Hissako Sumida, Prof ${ }^{\star}$
}

\section{Ahstract}

Introduction: Our previous studies have shown that periapical lesions (PLs) in rats cause systemic disorders such as increased tumor necrosis factor- $\alpha$ plasma levels, insulin resistance, and impairment in insulin signal transduction in muscle tissue. However, the mechanisms involved in these alterations are not fully understood. Under chronic inflammatory conditions such as obesity, it has been shown that the skeletal muscle is affected by inflammation, and the number of resident macrophages that are associated with impairments of insulin action and sensitivity is increased. This study aimed to investigate the presence of macrophages, activation of inflammatory pathways in muscle tissue, glycemia, and insulinemia of rats with PLs. Methods: Sixty Wistar rats were distributed into a control group; a group with $1 \mathrm{PL}(1 \mathrm{PL})$, which was induced in the right maxillary first molar; and a group with 4 PLs (4PL), which were induced in the right upper and lower first and second molars. We quantified macrophage content by immunohistochemistry for the $F 4 / 80$ protein. We evaluated Jun $\mathrm{N}$-terminal kinase and IKK $\alpha / \beta$ phosphorylation status in the muscle tissue by Western blotting. Serum levels of lipopolysaccharide (LPS) and HSP70 and plasma levels of glucose and insulin were assessed by using commercial kits. Results: The 1PL and 4PL groups showed increase in macrophage content, IKK $\alpha / \beta$, and Jun $\mathrm{N}$-terminal kinase phosphorylation status, serum LPS and HSP70 levels, and insulin resistance and no changes in glycemia and insulinemia compared with the control group. There was no difference in these parameters between the 1PL and 4PL groups. Conclusions: PLs promoted an increase in macrophage infiltration, activation of inflammatory pathways in mus- cle tissue, and serum concentrations of HSP70 and LPS in rats. The present study improves the knowledge on the impact of oral inflammations on the development of systemic alteration, which can induce insulin resistance. (J Endod 2017;43:982-988)

\section{Key Words}

Inflammation, insulin resistance, periapical lesion, systemic disorders

Clinir linical and experimental studies have shown an association between periapical lesions (PLs) and systemic disolrders such as diabetes and insulin resistance (1-4). Insulin resistance occurs when normal or

\section{Significance}

The present results suggest that oral inflammation may promote systemic disorders, resulting in alterations in concentrations of proinflammatory mediators in serum and in the metabolism of peripheral tissues such as muscle tissue, which has a key role in glucose homeostasis.

elevated levels of insulin cause a decreased biological response (5). Studies have reported a critical role of inflammation in the etiology of insulin resistance (6). In PLs, a local inflammatory process occurs in the dental pulp and periapical region (7). However, there is evidence that inflammatory marker levels are elevated in the serum of rats and humans with PLs, indicating that these markers can act systemically (8-10). Increased levels of inflammatory markers in the plasma are characterized as a state of chronic low-grade inflammation that is usually associated with obesity and can contribute to the development of insulin resistance (11). Furthermore, in obesity, there is evidence that muscle tissue is affected by an increase in the number of resident macrophages (12). F4/80 protein is known as a highly specific cell surface marker for murine macrophages. This protein is widely expressed in the majority of resident tissue macrophages (13). Macrophages express toll-like receptor 4 (TLR4) that can induce inflammatory responses by activating innate immunity, thereby contributing to the pathogenesis of insulin resistance (14). Studies show that macrophages can be activated by several factors including cytokines and bacterial endotoxins such as lipopolysaccharide (LPS) (15). LPS is a cell membrane component of gram-negative bacteria and plays a key role in activation of inflammation and periapical bone resorption (16). Another

From the *Programa de Pós-Graduação Multicêntrico em Ciências Fisiológicas-SBFis, Department of Basic Sciences, ${ }^{\dagger}$ Department of Restorative Dentistry, ${ }^{\S}$ Department of Child and Social Dentistry, and "Department of Basic Sciences, São Paulo State University (Unesp), School of Dentistry, Araçatuba; and ₹São Paulo State University (Unesp), Institute of Biosciences, Botucatu, Brazil.

Address requests for reprints to Dr Fernando Yamamoto Chiba, Dental School of Araçatuba, UNESP, University Estadual Paulista, R. José Bonifácio, 1193, CEP 16015050, Araçatuba, SP, Brazil. E-mail address: fernando_chiba@hotmail.com

0099-2399/\$ - see front matter

Copyright (C) 2017 American Association of Endodontists.

http://dx.doi.org/10.1016/j.joen.2017.01.030 
mediator that plays an active role in the modulation of inflammation is heat shock protein (HSP) 70, which presents an anti-inflammatory effect in the intracellular environment (17) and a proinflammatory effect in the extracellular environment (18) that may contribute to the development of insulin resistance (19).

Our previous studies have shown that PL in rats causes insulin resistance and impairs insulin signal transduction in muscle tissue $(3,4)$. However, the mechanisms involved are not fully understood. Because PL promotes an inflammatory state similar to that in obesity, this study aimed to investigate the presence of infiltrated macrophages by detection of $\mathrm{F} 4 / 80$ protein and to evaluate inflammatory pathways related to inhibition of insulin signal transduction in muscle tissues of rats with 1 or 4 teeth with PL. In addition, we quantified serum HSP70 and LPS levels, which are possible inducers of insulin resistance. The results of this study may help understand the interrelationship between local inflammation and systemic disorders.

\section{Materials and Methods}

Sixty male Wistar albino rats weighing 250-280 g were used in the study. The animals were housed with a 12-hour light/dark cycle (lights on at 7:00 $\mathrm{Am})$ in temperature-controlled rooms $\left(23^{\circ} \pm 2^{\circ} \mathrm{C}\right)$ and were given access to water and food (23\% from protein, $4 \%$ fat, and $58 \%$ from carbohydrate) (Presence; Paulínia, Sao Paulo, Brazil) ad libitum. All experiments were approved by the local ethics committee according to protocol number $00745-2015$.

\section{Animal Preparation and Death}

The 60 animals were randomly distributed into 3 groups (Fig. 1), with 20 animals in each group: a control group (CN), a group with 1 periapical lesion (1PL), and a group with 4 periapical lesions (4PL).
The rats were anesthetized by intraperitoneal injection with ketamine (Ketamina Agener; Embu Guacu, SP, Brazil) and xylazine (Dorcipec; Vallee, Montes Claros, MG, Brazil). To induce PLs, dental pulps of the right maxillary first molar (1PL) and of the right upper and lower first and second molars (4PL) were exposed to the oral environment on the occlusal surface by using a surgical round bur (Long Neck Maillefer; Dentsply, Petropolis, RJ, Brazil). For 30 days, body weight per rat was measured weekly. At 30 days after pulp exposure, the rats $(n=9)$ were subjected to a 14-hour fast, followed by anesthetization via intraperitoneal injection with sodium thiopental (Thiopentax; Cristalia, Itapira, SP, Brazil), and blood was collected. Subsequently, the animals $(n=9)$ were killed via overdose of the same anesthetic, and jaws were removed and fixed for 48 hours in $4 \%$ formaldehyde (Sigma-Aldrich, St Louis, MO) diluted in phosphate-buffered saline (PBS) (pH 7.4, $0.1 \mathrm{~mol} / \mathrm{L}$; Sigma-Aldrich). Gastrocnemius skeletal muscle was collected from 6 other animals to evaluate Jun N-terminal kinase (JNK) and IKK $\alpha / \beta$ phosphorylation status by Western blotting method. For immunohistochemical analysis of F4/80, the other animals $(n=5)$ were perfused as described by Da Silva et al (20). Immediately after the perfusion, the gastrocnemius muscle was post-fixed for 4 hours in $4 \%$ formaldehyde diluted in PBS.

\section{Histologic Processing of the Rat Jaws}

The right maxillae and mandible were decalcified in ethylenediamine tetraacetic acid (10\%; Sigma-Aldrich) for 60 days, processed in conventional manner, and embedded in paraffin. The parasagittal histologic sections of right maxillae first molar (1PL) or right upper and lower first and second molars (4PL) were obtained in microtome (RM2155; Leica Microsystems, BD, Wetzlar, Germany) with $4-\mu$ m-thick sections. The sections were stained with hematoxylin-eosin and

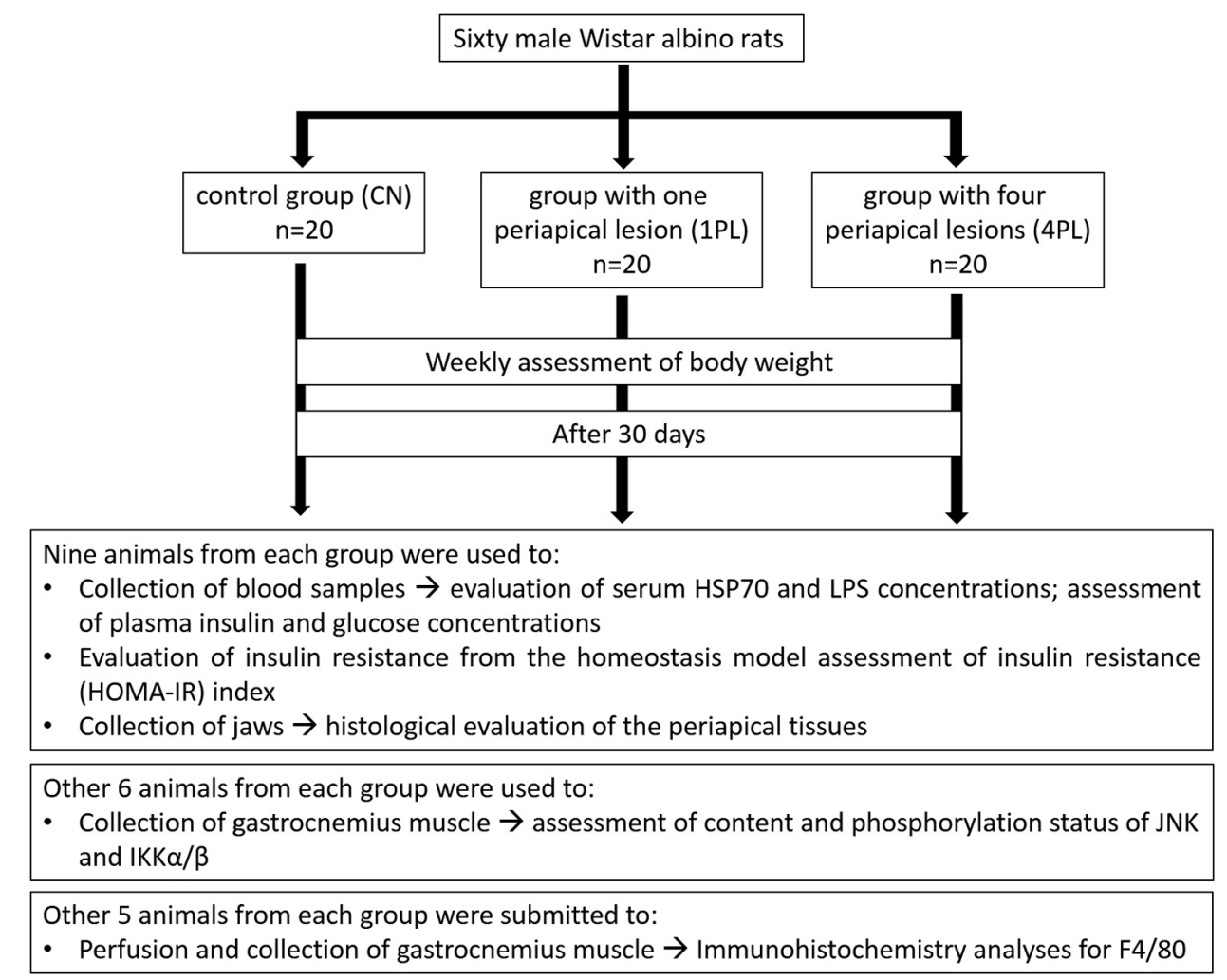

Figure 1. Flowchart showing step-by-step distribution of animals of $\mathrm{CN}$ group, 1PL group, and 4PL group to collection of blood samples, gastrocnemius muscle, and jaws used in the experiments. 

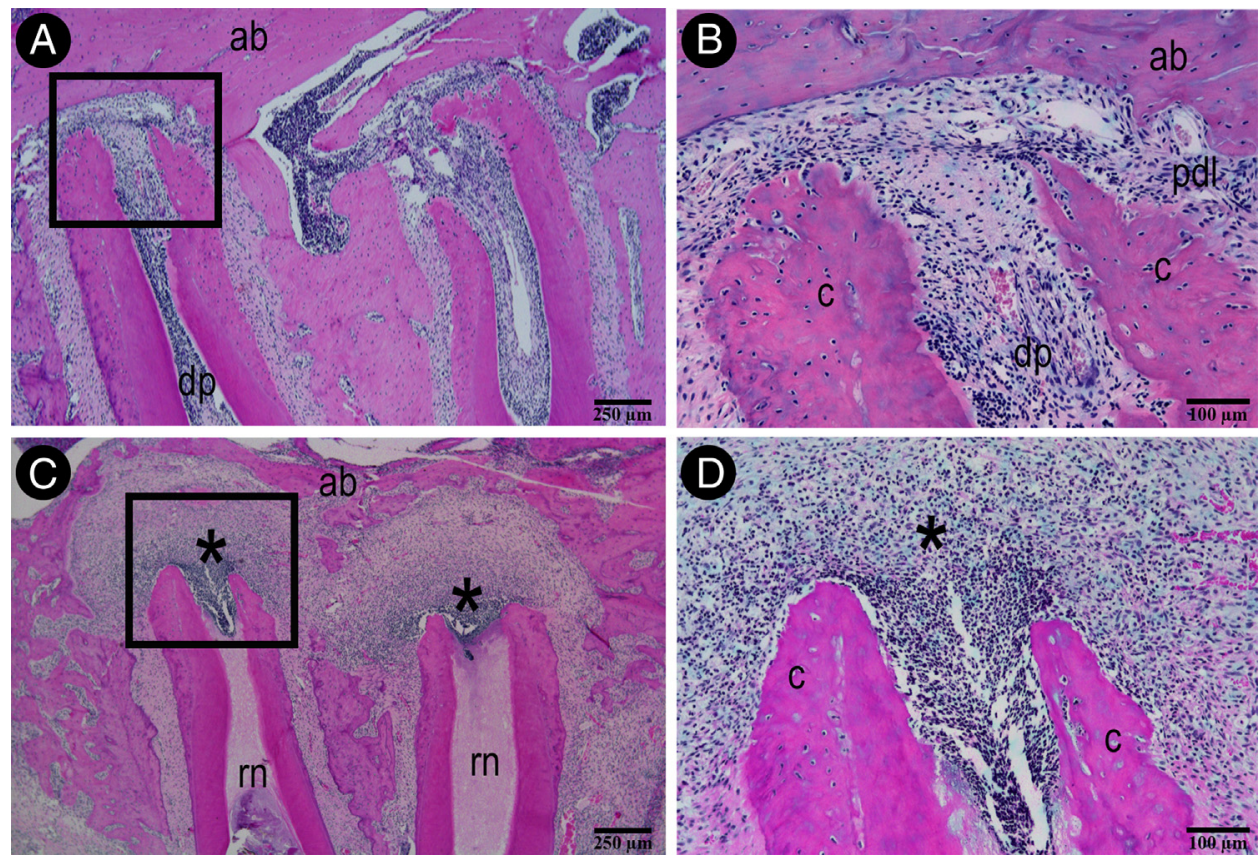

Figure 2. Photomicrography in light microscopy of histologic section of rat maxillae stained with hematoxylin-eosin in CN $(A$ and $B)$ and PL $(C$ and $D)$ groups. CN showed normal-appearing dental tissues. PL showed intense inflammatory infiltrate, predominantly mononucleate (asterisk). ab, alveolar bone; c, cement; dp, dental pulp; pdl, periodontal ligament; rn, remnants of pulp necrosis. Original magnification: $(A$ and $C), \times 50 ;(B$ and $D), \times 250$. Scale bars: $(A$ and $C)$, $250 \mu \mathrm{m} ;(B$ and $D), 100 \mu \mathrm{m}$.

analyzed by brightfield microscopy (Axio Lab A1; Carl Zeiss, TH, Oberkochen, Germany) to study the dental pulp and periapical region.

\section{Immunohistochemistry for F4/80 in Muscle}

The right gastrocnemius muscle was cryoprotected in $30 \% \mathrm{su}-$ crose (overnight) at $4^{\circ} \mathrm{C}$. Transversal histologic sections with $30-\mu \mathrm{m}$ thickness were obtained in a cryostat device (CM1850UV; Leica, HE, Germany) and collected on histologic slides. The sections were washed in PBS (0.1 mol/L, pH 7.4; Sigma-Aldrich), and nonspecific binding sites were blocked with PBS containing 3\% bovine albumin serum (Sigma-Aldrich) overnight at $4^{\circ} \mathrm{C}$. Later, the muscle sections were incubated in a solution of Triton X-100 (0.3\%; Sigma-Aldrich), normal donkey serum (Sigma-Aldrich), and rabbit polyclonal anti-F4-80 antibody (Santa Cruz Biotechnology, Santa Cruz, CA) overnight at $4^{\circ} \mathrm{C}$. Subsequently, the sections were incubated in solution with biotinylated secondary goat anti-rabbit antibody (Santa Cruz Biotechnology) for 2 hours at room temperature, rinsed in PBS, incubated in streptavidin conjugated with Cy3 (1:500; Jackson Immunoresearch, West Grove, PA) at room temperature for 1 hour, rinsed again in PBS, and coverslipped with buffered glycerol solution. The sections were imaged under a confocal laser scanning microscope (Leica TCS SP5 Confocal; Leica). For immunohistochemistry analyses, each image (32 per group) was acquired with $\times 40$ objective, and the cells that showed immunofluorescence for $\mathrm{F} 4 / 80$ (red fluorescence) were counted manually by using Image J (National Institutes of Health, Bethesda, MD) for the number of immunolabeled cells in relationship to the total number of cells per visual field.

\section{Assessment of Content and Phosphorylation Status of JNK and IKK $\alpha / \beta$}

Samples from the gastrocnemius muscle were collected from 6 animals from each group. Tissue samples were prepared according to the method described by Carvalho et al (21) and subjected to Western blotting for quantification of content and phosphorylation status of JNK and IKK $\alpha / \beta$ by using antibodies against $\mathrm{p}-\mathrm{IKK} \alpha / \beta$, p-JNK, IKK $\alpha / \beta$, and JNK (Santa Cruz Biotechnology Inc). Immunoreactive bands were detected by autoradiography using a chemiluminescent substrate system (GE Healthcare, Buckinghamshire, UK) according to the manufacturer's instructions. Quantitative analysis of the blots was conducted by using Scion Image software (Scion Image- Release Beta 3b; National Institutes of Health).

\section{Serum HSP70 and LPS Concentrations}

To determine the HSP70 and LPS serum concentrations, blood samples were collected $(n=9)$ from the inferior vena cava after the rats had fasted overnight for 8-12 hours. The blood samples were centrifuged immediately after collection at $1800 \mathrm{~g}$ for 15 minutes at $4^{\circ} \mathrm{C}$ to obtain serum. For quantification of serum LPS concentration, samples were diluted $1 / 40$ to $1 / 100$ and heated for 10 minutes at $70^{\circ} \mathrm{C}$. LPS was quantified by using a commercially available Pierce LAL Chromogenic Endotoxin Quantitation Kit (Thermo Scientific, Rockford, IL) according to the manufacturer's protocol. Circulating HSP70 was assessed by using EKS-715 high-sensitivity HSP70 kit according to the manufacturer's instructions (Enzo Life Sciences, Lausen, Switzerland).

\section{Glycemia, Insulinemia, and Homeostatic Model Assessment of Insulin Resistance Index}

Blood samples were collected from the inferior vena cava to measure plasma glucose and insulin concentrations. After centrifugation, supernatant was collected and maintained at $-80^{\circ} \mathrm{C}$ until use. Plasma samples from 9 animals per group were used for determination of glycemia by the oxidase glucose method (Enzymatic glucose; ANALISA Diagnóstica, Belo Horizonte, MG, Brazil) and of insulinemia by 

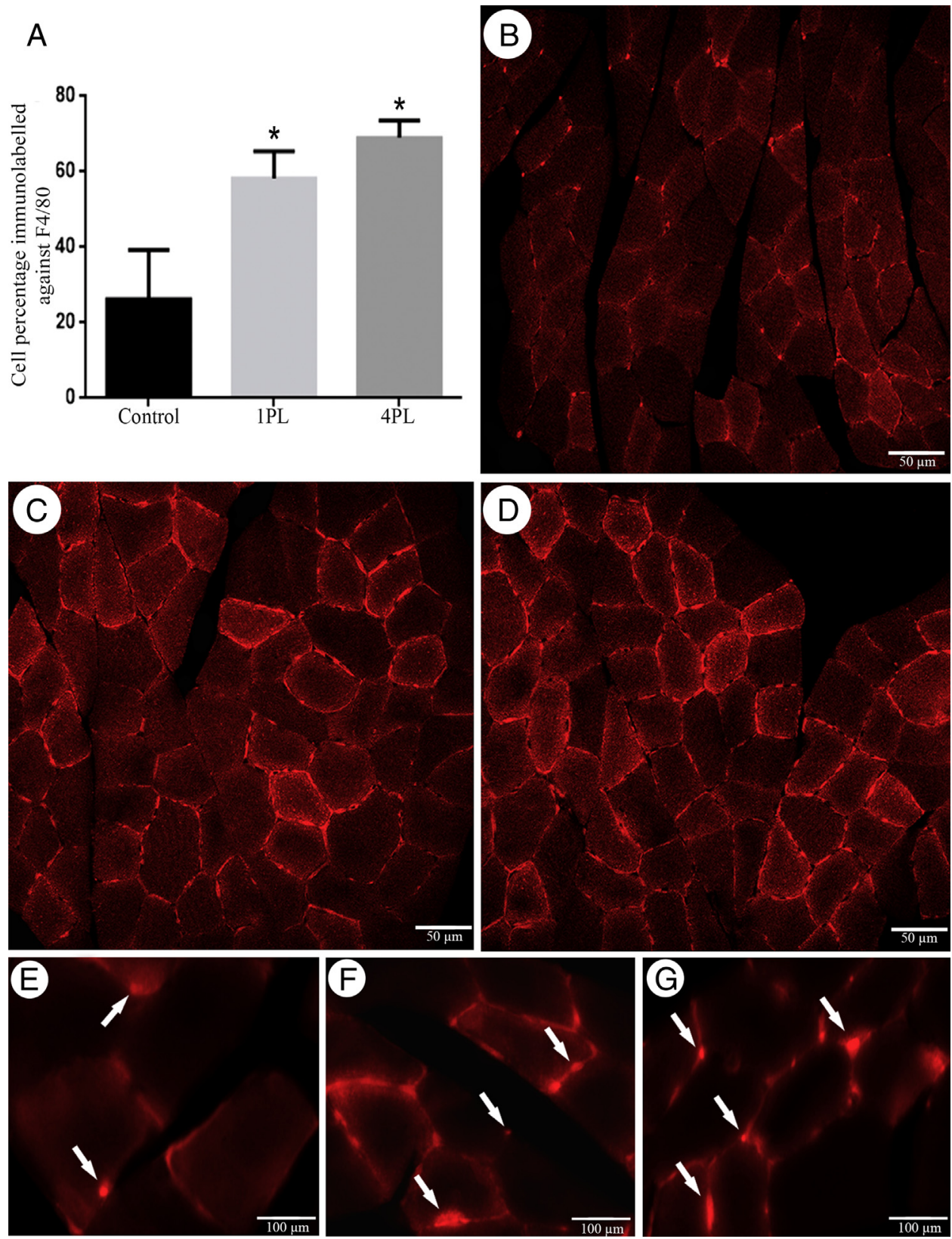

Figure 3. Immunofluorescence for F4/80 (red) in gastrocnemius muscle tissue from CN group, 1PL group, and 4PL group. (A) Percentage of immunolabeled cells for F4/80 in CN, 1PL, and 4PL groups. The cell photomicrographs, obtained from immunohistochemical analyses for F4/80 (red fluorescence) under confocal laser scanning microscope, represent the following groups: $\mathrm{CN}(B$ and $E), 1 \mathrm{PL}(C$ and $F)$, and 4PL $(D$ and $G)$ groups. Arrows indicate cells F4/80+ located around the muscular fibers. Values are presented as mean \pm SEM $(n=5) . * P<.05$ compared with $\mathrm{CN}$ group.

radioimmunologic assay (Coat-A-Count; DPC Diagnostic Products, Los Angeles, CA). Insulin resistance was evaluated by homeostasis model assessment of insulin resistance (HOMA-IR) index, calculated by the formula: HOMA-IR $=$ fasting glycemia $(\mathrm{mmol} / \mathrm{L}) \times$ fasting insulinemia $(\mu \mathrm{IU} / \mathrm{mL}) / 22.5(22)$.

\section{Statistical Analyses}

The normality of the data set was verified for all analyses. Analysis of variance was performed, followed by Tukey post hoc test $(P<.05)$. Data were expressed as the mean \pm standard error of the mean (SEM), and data analysis was performed with a statistical program (Graph Pad Prism version 6.0; GraphPad Software Inc, San Diego, CA).

\section{Results}

\section{Assessment of Body Weight of the Rats}

We found no difference in the body weights evaluated on the first, fifteenth, and thirtieth day after induction of PL among the groups.

\section{Histologic Evaluation of the Periapical Tissues}

In the $\mathrm{CN}$ group, the periapical tissue showed characteristics consistent with a normal aspect (Fig. $2 A$ and $B$ ). In both PL groups we observed the formation of PL in teeth subjected to exposure of the pulp to the oral environment. The lesions showed osteolysis and were filled with inflammatory infiltrate, predominantly mononucleate (Fig. $2 C$ and $D$ ). 

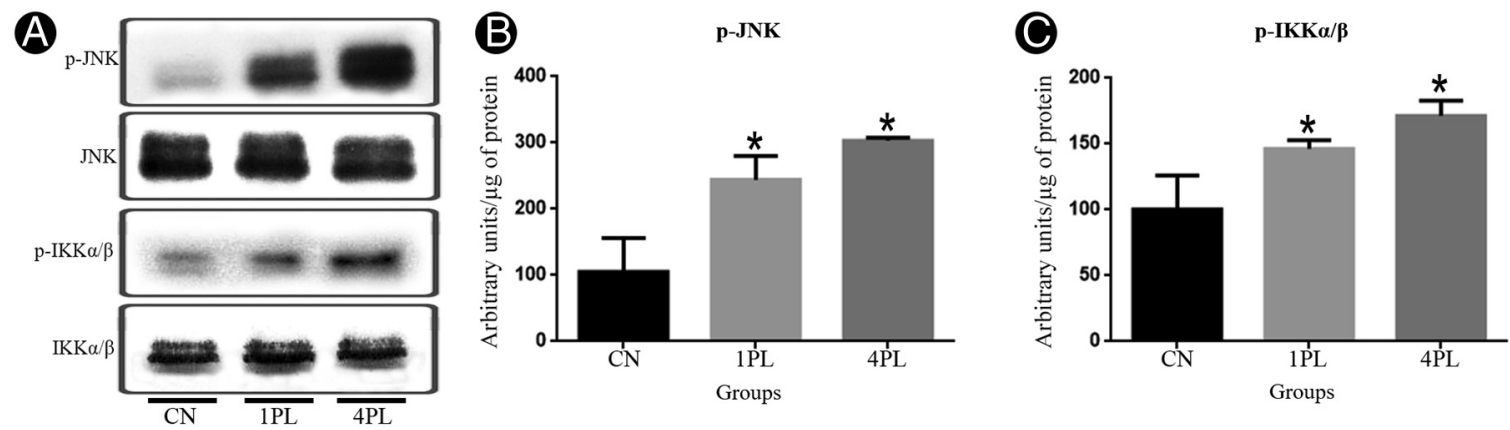

Figure 4. Evaluation of JNK and IKK $\alpha / \beta$ phosphorylation status in gastrocnemius muscle (GM). (A) Typical autoradiography: equal amounts of protein (185 $\mu \mathrm{g}$ ) were subjected to sodium dodecylsulfate-polyacrylamide gel electrophoresis; JNK and IKK $\alpha / \beta$ total content were used as controls. ( $B$ and $C$ ) Values of JNK and IKK $\alpha / \beta$ phosphorylation status expressed in arbitrary units are presented as mean \pm SEM $(n=6)$, respectively. $* P<.05$ compared with CN group.

\section{Immunohistochemical Analysis of F4/80}

The immunohistochemical results revealed the presence of immunolabeled cells for F4/80 in control, 1PL, and 4PL groups (Fig. 3B-G). Increase in the number of immunolabeled cells for $F 4 / 80$ around the fibers of gastrocnemius muscle in both the 1PL and 4PL groups (Fig. $3 C, D, F$, and $G$ ) were observed when compared with that in the $\mathrm{CN}$ group. We observed significant differences between $\mathrm{CN}$ and 1PL $(P<.05)$ and between $\mathrm{CN}$ and 4PL $(P<.05)$. However, no statistical difference was observed between the 1PL and 4PL groups (Fig. $3 A$ ).

\section{Assessment of Content and Phosphorylation Status of JNK and IKK $\alpha / \beta$}

We observed an increase in JNK and IKK $\alpha / \beta$ phosphorylation status $(P<.05)$ in the 1PL and 4PL groups compared with that in the $\mathrm{CN}$ group (Fig. $4 A-C$ ). We found no difference in these parameters between 1PL and 4PL groups.

\section{Serum HSP70 and LPS Concentrations}

We found higher levels of LPS and HSP70 in 1PL and 4PL groups compared with that in the $\mathrm{CN}$ group (Table 1). We found no difference in these parameters between 1PL and 4PL groups.

\section{Glycemia, Insulinemia, and HOMA-IR Index}

The glycemia, insulinemia, and HOMA-IR data are shown in Table 2. There are no differences in blood glucose and insulin levels between CN and PL groups. However, the HOMA-IR calculated from these values was significantly higher in 1PL and 4PL groups than in the $\mathrm{CN}$ group. We found no difference in these parameters between 1PL and 4PL groups.

\section{Discussion}

The present study demonstrates that rats with 1 or 4 teeth with PL showed increase in the number of infiltrating macrophages, IKK $\alpha / \beta$ and JNK phosphorylation status in the muscle tissue, and serum LPS and HSP70 levels, and insulin resistance and no changes in glycemia and insulinemia compared with the $\mathrm{CN}$ rats.
It was observed that PL1 and PL4 groups showed insulin resistance as demonstrated by the HOMA-IR index. These data are in agreement with previous studies that showed that rats with PL exhibit insulin resistance assessed both by HOMA-IR index and by insulin tolerance test after 30 days of exposure to PL (2-4). Studies have shown that macrophage contents in skeletal muscle of obese animals and humans are correlated with impairment in insulin sensitivity $(12,23)$. The present study demonstrates that 1PL and 4PL groups showed higher macrophage content in the muscle tissue compared with the $\mathrm{CN}$ group (Fig. 3). Recent study (24) showed that only 5 days of high-fat diet consumption can promote an increase in RNA expression of macrophage markers and a decrease in expression of glucose transporter type 4 in skeletal muscle in healthy lean men, probably because the high-fat diet leads to inflammatory processes in the muscle, which contributes to the development of insulin resistance by releasing proinflammatory cytokines. Moreover, Parsoturis et al (25) observed that increased macrophage infiltration in muscle tissue correlated with insulin resistance in patients with diabetes, regardless of body mass index. These data corroborate our results of increased macrophage infiltration in the skeletal muscle of rats with PL in the absence of obesity (Table 3), highlighting the key role of macrophages in insulin resistance etiology.

LPS and HPS70 play an active role in inflammation, which is closely related to development of insulin resistance. For this reason, we quantified the levels of serum LPS and HSP70 to verify the relationship of these mediators with insulin resistance, which was observed in previous studies performed in rats with PL. Our results show that the levels of serum LPS and HSP70 increased in the 1PL and 4PL groups compared with those in the control group (Table 1). Studies suggest that chronic elevation of circulating LPS levels has a key role in the pathogenesis of insulin resistance $(26,27)$. This is probably due to the interaction of LPS with TLR4, which results in activation of inflammatory pathways and negatively affects the insulin signal (27).

Recent study demonstrated that HSP gene expression is increased in periapical granulomas compared with that in healthy periodontal tissue (28). Thus, the results of the present study indicate that a local increase in HSP gene expression can result in increased concentration of this protein in the serum. Notably, studies showed increase in levels of

TABLE 1. Concentrations of Serum LPS and HSP70 in CN, 1PL, and 4PL Groups

\begin{tabular}{lccc}
\hline & CN & 1 PL & $4 P L$ \\
\hline LPS (endotoxin unit/mL) & $0.40 \pm 0.07$ & $1.40 \pm 0.39 *$ & $1.53 \pm 0.30^{*}$ \\
HSP70 (ng/mL) & $0.28 \pm 0.01$ & $0.47 \pm 0.06^{*}$ & $0.53 \pm 0.05^{*}$ \\
\hline
\end{tabular}

Values are presented as mean \pm SEM $(n=9)$.

${ }^{*} P<.05$ compared with $\mathrm{CN}$ group. 
Basic Research-Biology

TABLE 2. Glycemia, Insulinemia, and HOMA-IR Index in CN, 1PL, and 4PL Groups

\begin{tabular}{lrrr}
\hline & \multicolumn{1}{c}{ CN } & \multicolumn{1}{c}{ 1PL } & 4PL \\
\hline Glycemia $(\mathrm{mmol} / \mathrm{L})$ & $6.94 \pm 0.33$ & $6.74 \pm 0.14$ & $6.77 \pm 0.21$ \\
Insulinemia $(\mu \mathrm{IU} / \mathrm{mL})$ & $19.14 \pm 6.35$ & $29.21 \pm 4.21$ & $31.80 \pm 3.12$ \\
HOMA-IR & $5.42 \pm 1.86$ & $10.18 \pm 0.77^{*}$ & $10.31 \pm 0.97^{*}$ \\
\hline
\end{tabular}

Values are presented as mean \pm SEM $(\mathrm{n}=9)$.

${ }^{*} P<.05$ compared with $\mathrm{CN}$ group.

plasma HSP70 in patients with diabetes $(29,30)$ and a positive correlation of this marker with other chronic diseases (31). Furthermore, it has been demonstrated that HSP70 can activate TLR4, indicating a possible involvement of this protein in the development of insulin resistance (32). These data indicate that LPS and HSP70 have a fundamental role in the association between insulin resistance and PL.

To assess the molecular mechanisms involved in insulin resistance in PL, we evaluated the phosphorylation status of JNK and IKK in the rat muscle tissue. Our results demonstrate that the 1PL and 4PL groups showed higher phosphorylation of these proteins compared with the $\mathrm{CN}$ group. The findings of the present study are in agreement with studies that observed the relationship between insulin resistance and JNK and IKK activation $(33,34)$. Kaneto et al $(35)$ demonstrated that treatment of diabetic rats with JNK inhibitors improved insulin sensitivity of the animals. Similarly, the studies of Yuan et al (36) and Hundal et al (37) showed that IKK inhibition by administration of salicylate improves insulin action in obese diabetic humans and rats, respectively. Therefore, because insulin resistance is related to local and systemic inflammatory processes, it is possible that the mechanisms involved in the development of insulin resistance in rats with PL involve a crosstalk between immunoinflammatory response in PL and the skeletal muscle tissue.

In the present study, rats with 1 and 4 teeth with PL were used to simulate the conditions observed in the population because epidemiologic studies performed in different populations demonstrated wide variation in the number of teeth with PL in the same person $(38,39)$. Recently, Cintra et al (10) evaluated whether apical periodontitis in a single tooth or in 4 teeth promotes changes in levels of inflammatory mediators in the serum and verified that levels of serum interleukin (IL)-6, IL-17, IL-23, and tumor necrosis- $\alpha$ increased only in rats with apical periodontitis in 4 teeth compared with those in control rats. However, a previous study showed that the presence of only 1 tooth with PL is enough to promote an increase in concentrations of plasma tumor necrosis factor- $\alpha$ (2). In our study, we also observed that only one focus of PL was able to promote an increase in the concentrations of serum HSP70 and LPS, which could induce systemic alterations related to increase in activation of inflammatory pathways.

This is the first study to demonstrate the existence of a crosstalk between PL and muscle tissue. Our results showed that 30 days of exposure to PL promoted macrophage infiltration, activation of inflammatory pathways in muscle tissue, and increased concentrations of serum HSP70 and LPS in rats. The present results suggest that oral inflammation may promote systemic disorders, resulting in alteration in concentrations of proinflammatory mediators in the serum and in the

TABLE 3. Body Weight (g) of the CN and PL Groups of Rats

\begin{tabular}{lccc}
\hline Groups & First day & Fifteenth day & Thirtieth day \\
\hline CN & $266.4 \pm 2.08$ & $336.1 \pm 1.93$ & $405.9 \pm 2.13$ \\
$1 P L$ & $266.9 \pm 1.78$ & $337.6 \pm 1.63$ & $408.1 \pm 1.87$ \\
$4 \mathrm{PL}$ & $266.4 \pm 1.81$ & $336.4 \pm 1.81$ & $407.0 \pm 1.89$ \\
\hline
\end{tabular}

Values are presented as mean \pm SEM $(\mathrm{n}=20)$. metabolism of peripheral tissues such as muscle tissue, which has a key role in glucose homeostasis.

The present study improves the knowledge on the impact of oral inflammations on development of systemic alterations that can induce insulin resistance, thus contributing to the development of new therapeutic approaches.

\section{Acknowledgments}

The authors thank Shelly Favorito de Carvalho from Electron Microscopy Center (EMC) of the Institute of Biosciences of Botucatu, UNESP, for assistance in the capture of confocal microscopy images for this manuscript.

This study was supported by a grant (2014/17619-6) from the

São Paulo Research Foundation (FAPESP), São Paulo, SP, Brazil.

The authors deny any conflicts of interest related to this study.

\section{References}

1. López-López J, Jané-Salas E, Estrugo-Devesa A, et al. Periapical and endodontic status of type 2 diabetic patients in Catalonia, Spain: a cross-sectional study. J Endod 2011;37:598-601.

2. Astolphi RD, Curbete MM, Colombo NH, et al. Periapical lesions decrease insulin signal and cause insulin resistance. J Endod 2013;39:648-52.

3. Astolphi RD, Curbete MM, Chiba FY, et al. Periapical lesions decrease insulin signaling in rat skeletal muscle. J Endod 2015;41:1305-10.

4. Pereira RF, de Oliveira da Mota MS, de Lima Coutinho Mattera MS, et al. Periapical lesions decrease Akt serine phosphorylation and plasma membrane GLUT4 content in rat skeletal muscle. Clin Oral Investig 2016;20:1625-30.

5. Wilcox G. Insulin and insulin resistance. Clin Biochem Rev 2005;26:19-39.

6. Coope A, Torsoni AS, Velloso LA. Mechanisms in endocrinology: metabolic and inflammatory pathways on the pathogenesis of type 2 diabetes. Eur J Endocrinol 2016; 174:r175-87.

7. Fouad AF, Acosta AW. Periapical lesion progression and cytokine expression in an LPS hyporesponsive model. Int Endod J 2001;34:506-13.

8. Gomes MS, Blattner TC, Sant'Ana Filho M, et al. Can apical periodontitis modify systemic levels of inflammatory markers? a systematic review and meta-analysis. J Endod 2013;39:1205-17.

9. Zhang H, Bain JL, Caskey CP, et al. Effects of gender on serum biomarkers of systemic inflammation coincident to experimentally-induced periapical lesions. Arch Oral Biol 2011;56:168-76.

10. Cintra LT, Samuel RO, Azuma MM, et al. Multiple apical periodontitis influences serum levels of cytokines and nitric oxide. J Endod 2016;42:747-51.

11. Heilbronn LK, Campbell LV. Adipose tissue macrophages, low grade inflammation and insulin resistance in human obesity. Curr Pharm Des 2008;14:1225-30.

12. Fink LN, Costford SR, Lee YS, et al. Pro-inflammatory macrophages increase in skeletal muscle of high fat-fed mice and correlate with metabolic risk markers in humans. Obesity (Silver Spring) 2014;22:747-57.

13. Lin $H$, Faunce DE, Stacey $M$, et al. The macrophage F4/80 receptor is required for the induction of antigen-specific efferent regulatory T cells in peripheral tolerance. JEM 2005:201:1615-25.

14. Shi H, Kokoeva MV, Inouye K, et al. TLR4 links innate immunity and fatty acidinduced insulin resistance. J Clin Invest 2006;116:3015-25.

15. Metzger Z. Macrophages in periapical lesions. Endod Dent Traumatol 2000;16:1-8.

16. Narayanan LL, Vaishnavi C. Endodontic microbiology. J Conserv Dent 2010;13: 233-9.

17. Ran R, Lu A, Zhang L, et al. Hsp 70 promotes TNF-mediated apoptosis by binding IKK gamma and impairing NF-kappa B survival signaling. Genes Dev 2004;18:1466-81.

18. Asea A, Kraeft SK, Kurt-Jones EA, et al. HSP70 stimulates cytokine production through a CD14-dependent pathway, demonstrating its dual role as a chaperone and cytokine. Nat Med 2000;6:435-42. 
19. Krause M, Heck TG, Bittencourt A, et al. The chaperone balance hypothesis: the importance of the extracellular to intracellular HSP70 ratio to inflammationdriven type 2 diabetes, the effect of exercise, and the implications for clinical management. Mediators Inflamm 2015;2015:249205.

20. Da Silva AV, Torres KR, Haemmerle CA, et al. The Edinger-Westphal nucleus II: hypothalamic afferents in the rat. J Chem Neuroanat 2013;54:5-19.

21. Carvalho CR, Brenelli SL, Silva AC, et al. Effect of aging on insulin receptor, insulin receptor substrate-1, and phosphatidylinositol 3-kinase in liver and muscle of rats. Endocrinology 1996;137:151-9.

22. Bonora E, Targher G, Alberiche M, et al. Homeostasis model assessment closely mirrors the glucose clamp technique in the assessment of insulin sensitivity: studies in subjects with various degrees of glucose tolerance and insulin sensitivity. Diabetes Care 2000;23:57-63.

23. Varma V, Yao-Borengasser A, Rasouli N, et al. Muscle inflammatory response and insulin resistance: synergistic interaction between macrophages and fatty acids leads to impaired insulin action. Am J Physiol Endocrinol Metab 2009;296:E1300-10.

24. Boon MR, Bakker LE, Haks MC, et al. Short-term high-fat diet increases macrophage markers in skeletal muscle accompanied by impaired insulin signalling in healthy male subjects. Clin Sci (Lond) 2015;128:143-51.

25. Patsouris D, Cao JJ, Vial G, et al. Insulin resistance is associated with MCP1-mediated macrophage accumulation in skeletal muscle in mice and humans. PLoS One 2014; 9:e110653.

26. Watanabe K, Iizuka T, Adeleke A, et al. Involvement of toll-like receptor 4 in alveolar bone loss and glucose homeostasis in experimental periodontitis. J Periodontal Res 2011;46:21-30.

27. Liang H, Hussey SE, Sanchez-Avila A, et al. Effect of lipopolysaccharide on inflammation and insulin action in human muscle. PLoS One 2013;8:e63983.

28. Goodman SC, Letra A, Dorn S, et al. Expression of heat shock proteins in periapical granulomas. J Endod 2014;40:830-6.
29. Nakhjavani M, Morteza A, Khajeali L, et al. Increased serum HSP70 levels are associated with the duration of diabetes. Cell Stress Chaperones 2010;15:956-94.

30. Hunter-Lavin C, Hudson PR, Mukherjee S, et al. Folate supplementation reduces serum hsp70 levels in patients with type 2 diabetes. Cell Stress Chaperones 2004; 9:344-9.

31. Qu B, Jia Y, Liu Y, et al. The detection and role of heat shock protein 70 in various nondisease conditions and disease conditions: a literature review. Cell Stress Chaperones 2015;20:885-92.

32. Asea A, Rehli M, Kabingu E, et al. Novel signal transduction pathway utilized by extracellular HSP70: role of toll-like receptor (TLR) 2 and TLR4. J Biol Chem 2002;277: 15028-34.

33. Jiao P, Ma J, Feng B, et al. FFA-induced adipocyte inflammation and insulin resistance: involvement of ER stress and IKK $\beta$ pathways. Obesity (Silver Spring) 2011; 19:483-91.

34. Pal M, Febbraio MA, Lancaster GI. The roles of c-Jun NH2-terminal kinases (JNKs) in obesity and insulin resistance. J Physiol 2016;594:267-79.

35. Kaneto H, Nakatani Y, Miyatsuka T, et al. Possible novel therapy for diabetes with cell-permeable JNK-inhibitory peptide. Nat Med 2004;10:1128-32.

36. Yuan M, Konstantopoulos N, Lee J, et al. Reversal of obesity- and diet-induced insulin resistance with salicylates or targeted disruption of Ikkbeta. Science 2001; 293:1673-7.

37. Hundal RS, Petersen KF, Mayerson AB, et al. Mechanism by which high-dose aspirin improves glucose metabolism in type 2 diabetes. J Clin Invest 2002;109:1321-6.

38. Huumonen S, Suominen AL, Vehkalahti MM. Prevalence of apical periodontitis in root filled teeth: findings from a nationwide survey in Finland. Int Endod J 2017; 50:229-36.

39. Marotta PS, Fontes TV, Armada L, et al. Type 2 diabetes mellitus and the prevalence of apical periodontitis and endodontic treatment in an adult Brazilian population. J Endod 2012;38:297-300. 